\title{
The use of hyperbaric oxygen therapy in the treatment of necrotizing soft tissue infections, compromised grafts and flaps, hidradenitis suppurativa, and pyoderma gangrenosum
}

\author{
Clare A. Goggins ${ }^{1}$, Amor Khachemoune ${ }^{2 \otimes}$
}

\begin{abstract}
Hyperbaric oxygen therapy (HBOT) is used in the management of a wide array of disease states, including acute thermal burn injuries, carbon monoxide poisoning, and decompression sickness, to name a few. Although HBOT is approved by the Undersea and Hyperbaric Medical Society for the treatment of only 14 conditions, it has been used "off-label" in the management of a variety of dermatological diseases. This review investigates the utilization of HBOT in dermatology and appraises the evidence behind its use. We focus on the role of HBOT in treating necrotizing soft tissue infections, compromised grafts and flaps, hidradenitis suppurativa, and pyoderma gangrenosum.
\end{abstract}

Keywords: hyperbaric oxygen, necrotizing soft tissue infections, necrotizing fasciitis, compromised grafts and flaps, pyoderma gangrenosum, hidradenitis suppurativa

Received: 21 September 2018 | Returned for modification: 19 November 2018 | Accepted: 3 January 2019

\section{Introduction}

Hyperbaric oxygen therapy (HBOT) is a treatment modality that employs oxygen at elevated atmospheric pressure. HBOT involves having a patient breathe in approximately 100\% oxygen while enclosed in a hyperbaric chamber set at a pressure greater than sea level ( $1 \mathrm{ATA}=101.325 \mathrm{kPa})(1)$. To achieve clinical benefit, the pressure within the chamber must be equal to or greater than 1.4 ATA (141.86 kPa) (1). The value of HBOT stems from four synergistic modalities. These include i) alleviation of hypoxia, ii) potentiation of ionizing radiation, iii) prevention of growth and toxin production by certain bacteria, and iv) maintenance of function of tissues at low temperatures (2).

\section{Historical perspective}

Literature on the use of HBOT initially emerged in the 1930 (1). During this decade, universities and navies across the globe began to experiment with using oxygen at elevated pressure to treat arterial gas embolism and decompression sickness (1). The first publication describing the use of HBOT to treat decompression sickness was published in 1937 in the United States Naval Medical Bulletin (3). By the 1940s, HBOT had been incorporated into standard treatment tables of the United States Navy (1). In 1960, Ite Boerema was the first to utilize HBOT for the treatment of gas gangrene (1).

\section{Current indications for the use of HBOT}

According to the Undersea and Hyperbaric Medical Society, HBOT is approved for the treatment of 14 disease states. These include air or gas embolism, clostridial myositis and myonecrosis (gas gangrene), carbon monoxide poisoning, compartment syndrome, crush injury, and other acute traumatic ischemias, necrotizing soft tissue infections (NSTIs), decompression sickness, arterial insufficiencies, intracranial abscess, severe anemia, refractory osteomyelitis, compromised grafts and flaps, delayed radiation injury (soft tissue and bony necrosis), acute thermal burn injury, and idiopathic sudden sensorineural hearing loss (1). Of these 14 conditions, NSTIs and compromised grafts and flaps are the most relevant to dermatology. However, HBOT has been used "offlabel” for the treatment of several other dermatological diseases.

\section{The use of HВОТ in dermatology}

HBOT has been used in the treatment of many dermatological conditions, including NSTIs, compromised grafts and flaps, poorly healing or recalcitrant ulcers (e.g., pyoderma gangrenosum, livedoid vasculopathy, and venous stasis), hidradenitis suppurativa (HS), toxic epidermal necrolysis, post-injection necrosis, calciphylaxis, Hansen's disease (leprosy), scleroderma, and brown recluse spider envenomation (4-6, 8-29). This review summarizes and appraises the evidence regarding the use of HBOT in the treatment of several dermatological diseases; specifically, NSTIs, compromised grafts and flaps, pyoderma gangrenosum, and HS (Table 1). We searched PubMed MEDLINE and Ovid MEDLINE using the keywords "hyperbaric oxygenation," "necrotizing soft tissue infections," "compromised grafts and flaps," "pyoderma gangrenosum," and "hidradenitis suppurativa."

\section{Necrotizing soft tissue infections}

HBOT was utilized as an adjunctive therapy for NSTIs as early as 1960 (1). In 2004, Wilkinson et al. published a retrospective cohort study evaluating the use of HBOT for the treatment of NSTIs (4). The primary endpoint used in the study was death during hospital stay, and the secondary endpoint was the need for limb amputation. A total of 44 NSTI cases were included in the study, of which 33 received HBOT in addition to standard management. The control group of 11 patients received only standard management. The median number of HBOT sessions was eight with a range of one to 30 , reflecting the fact that some patients received additional HBOT 
sessions even after their infection had been cleared to promote wound healing. Wilkinson et al. found that the addition of HBOT to standard therapy led to a decrease in the relative risk of death by $83 \%$, which corresponded to a number needed to treat of three. Of the 33 patients in the HBOT group, two died during their hospitalization. In comparison, of the 11 patients in the control group, four died during their hospitalization. The difference in survival between the groups was statistically significant $(p=0.02)(4)$.

In contrast, a retrospective review by George et al. that investigated the adjunctive use of HBOT for NSTIs found no statistically significant difference in the length of hospital stay, length of antibiotic use, or mortality rate between the HBOT and non-HBOT groups (5). A total of 78 patients were included in the study; of these, 48 received adjunctive HBOT therapy. Although the HBOT group had a lower mortality rate $(8.3 \%)$ compared to the nonHBOT group (13.3\%), this difference was not statistically significant $(p=0.48)(5)$.

A Cochrane systematic review published in 2015 aimed to review published evidence regarding the use of HBOT as an adjunctive therapy for patients with necrotizing fasciitis (6). The review sought to include published randomized trials and "pseudo-randomized" trials that compared the use of HBOT as an adjunctive treatment for NSTI to no adjunctive HBOT treatment. The HBOT intervention had to be provided in a mono-place or multi-place chamber set between 1.5 and 3 ATA. It had to last for 60 minutes or longer and occur at least once daily, with at least one treatment. Studies that utilized any antibiotic or surgical therapies in addi- tion to HBOT could be included if both the treatment and control groups received the same antibiotic and/or surgical therapy. The authors searched the Cochrane Central Register of Controlled Trials (CENTRAL), EMBASE Ovid (1980 to September 2014), MEDLINE Ovid (1966 to September 2014), the Database of Randomized Controlled Trials in Hyperbaric Medicine (from its inception to September 2014), and the Cumulative Index to Nursing and Allied Health Literature (CINAHL) Ovid (1982 to September 2014). The initial search revealed 906 hits, and, after duplicates were removed, the authors were left with 673 publications. However, upon viewing the abstracts of each of these publications, the authors found that none fit the inclusion criteria (6).

The authors concluded that there was insufficient clinical evidence to support or contest the utilization of HBOT in the treatment of necrotizing fasciitis (6). This Cochrane review importantly highlights the need for high-quality randomized controlled clinical trials investigating the use of HBOT as an adjunctive therapy for NSTI. In some places, HBOT is being provided as routine practice for the treatment of NSTI despite the lack of wellconducted trials demonstrating its efficacy (6). Although serious adverse effects with HBOT are rare, they have been reported (7). The most common side effects of HBOT are middle ear barotrauma and sinus/paranasal barotrauma, which are generally mild and self-limiting. The more worrisome adverse effects that have been reported with HBOT are pulmonary barotrauma and central nervous system oxygen toxicity (7). Given that HBOT does pose a risk of significant adverse effects, it deserves to be thoroughly exam-

Table 1 | Use of HBOT in four dermatological conditions.

Disease $\frac{\text { References }}{\text { Necrotizing soft tissue infections Wilkinson D, Doolette D. Hyperbaric oxygen treatment and survival from necrotizing soft tissue infection. Arch Surg. }}$ Necrotizing soft tissue infections Wilkinson D, Doolette D. Hyperbaric oxygen treatment and survival from necrotizing soft tissue infection. Arch Surg.
\[ 2004 ; 139: 1339-45 . \]

Levett D, Bennet MH, Millar I. Adjunctive hyperbaric oxygen for necrotizing fasciitis. Cochrane Database Syst Rev. 2015;1:CD007937.

George ME, Rueth NM, Skarda DE, Chipman JG, Quickel RR, Beilman GJ. Hyperbaric oxygen does not improve outcome in patients with necrotizing soft tissue infection. Surg Infect (Larchmt). 2009;10:21-8.

Compromised grafts and flaps Francis A, Baynosa RC. Hyperbaric oxygen therapy for the compromised graft or flap. Adv Wound Care. 2017;6:23-32. Larson JV, Steensma EA, Flikkema RM, Norman EM. The application of hyperbaric oxygen therapy in the management of compromised flaps. Undersea Hyperb Med. 2013;40:499-504.

Skeik N, Porten BR, Isaacson E, Seong J, Klosterman DL, Garberich RF, et al. Hyperbaric oxygen treatment outcome for different indications from a single center. Ann Vasc Surg. 2015;29:206-14.

Goldman RJ. Hyperbaric oxygen therapy for wound healing and limb salvage: a systematic review. PM R. 2009;1:47189.

Hidradenitis suppurativa Ozdemir Y, Uzun G, Mutluoglu M, Gulec B. Hyperbaric oxygen therapy for the management of postsurgical wounds in hidradenitis suppurativa. Am Surg. 2010;76:E237-8.

Yildiz H, Senol L, Ercan E, Bilgili ME, Karabudak AO. A prospective randomized controlled trial assessing the efficacy of adjunctive hyperbaric oxygen therapy in the treatment of hidradenitis suppurativa. Int J Dermatol. 2016;55:232-7. Sharon-Guidetti A, Ziv Y, Kummer E, Yogev R, Halevy A. Granulocyte-macrophage colony-stimulating factor for perianal hidradenitis suppurativa: report of a case. Dis Colon Rectum. 2006;49:682-4.

Wasserteil V, Bruce S, Sessoms SL, Guntupalli KK. Pyoderma gangrenosum treated with hyperbaric oxygen therapy. Int J Dermatol. 1992;31:594-6.

Niezgoda JA, Cabigas EB, Allen HK, Simanonok JP, Kindwall EP, Krumenauer J. Managing pyoderma gangrenosum: a synergistic approach combining surgical debridement, vacuum-assisted closure, and hyperbaric oxygen therapy.

Pyoderma gangrenosum Plast Reconstr Surg. 2006;117:24e-8e.

Vieira WA, Barbosa LR, Martin LM. Hyperbaric oxygen therapy as an adjuvant treatment for pyoderma gangrenosum. An Bras Dermatol. 2011;86:1193-6.

Tutrone WD, Green K, Weinberg JM, Caglar S, Clarke D. Pyoderma gangrenosum: dermatologic application of hyperbaric oxygen therapy. J Drugs Dermatol. 2007;6:1214-9.

Cabalag MS, Wasiak J, Lim SW, Raiola FB. Inpatient management of pyoderma gangrenosum: treatments, outcomes, and clinical implications. Ann Plast Surg. 2015;74:354-60.

Feitosa MR, Féres FO, Tamaki CM, Perazzoli C, Bernardes MV, Parra RS, et al. Adjunctive hyperbaric oxygen therapy promotes successful healing in patients with refractory Crohn's disease. Acta Cir Bras. 2016;31:19-23.

Altunay IK, Sezgin SA, Ileri U, Ekmekçi TR, Kuran I, Köslü A, et al. Atypical hemorrhagic bullous pyoderma gangrenosum. Int J Dermatol. 2001;40:327-9.

Callen JP, Case JD, Sager D. Chlorambucil-an effective corticosteroid-sparing therapy for pyoderma gangrenosum. J Am Acad Dermatol. 1989;21:515-9. 
ined in a randomized controlled clinical trial setting.

\section{Compromised grafts and flaps}

HBOT has also been used as an adjunctive treatment for compromised grafts and flaps. The standard treatment for a compromised graft or flap is local wound care, surgical debridement, and repeat reconstruction. HBOT increases oxygenation, improves fibroblast function, and promotes neovascularization; therefore, it has been used in addition to traditional therapies in cases of compromised grafts and flaps (8). A retrospective review published by Larson et al. in 2013 included 15 patients that had received HBOT for a compromised flap (9). In 11 patients (73.3\%), treatment success was achieved. Of these 11 patients, four $(36.4 \%)$ had complete healing of the compromised flap, and seven demonstrated (63.6\%) significant improvement (9). Another recent retrospective review by Skeik et al. examined the use of HBOT for a variety of conditions, including compromised grafts or flaps (10). Skeik et al. found that, of those patients with a compromised graft or flap, $75.7 \%$ had an improved outcome with HBOT. The average number of HBOT sessions that these patients received was 30 (10).

However, more high-quality studies investigating the use of HBOT for compromised grafts and flaps are needed. A systematic review by Goldman et al. included four case series and six case reports investigating the use of HBOT in compromised grafts and flaps (11). All 10 reported positive results with HBOT; however, of these 10 reports, nine had a low strength of evidence and one had a moderate strength of evidence. For this reason, Goldman et al. concluded that there was only "a low to moderate level of evidence" that HBOT can improve the success rate of compromised grafts and flaps (11).

\section{Hidradenitis suppurativa}

The use of HBOT as an adjunctive therapy to the surgical management of HS has also been examined. A case by Ozdemir et al. described a patient with severe HS that underwent total surgical excision with flap closure complicated by flap necrosis and local wound infection (12). After not responding to 2 weeks of attentive wound care and antibiotic therapy, HBOT was initiated. The patient underwent a total of 15 HBOT sessions over the course of 3 weeks. Each session was 90 minutes long at 2.0 ATA. Soon after starting HBOT, the infection cleared and healthy granulation tissue was noted at the base of the wound. At a 3-month follow-up, the patient's wound had completely healed without complications (12).

In 2016, Yildiz et al. published a prospective randomized control trial investigating the effect of adjunctive HBOT for the antibiotic treatment of HS (13). A total of 43 patients were randomly divided into a treatment group and a control group. Both groups were started on clindamycin $300 \mathrm{mg}$ twice daily and rifampicin $300 \mathrm{mg}$ twice daily, and were followed for a total of 10 weeks. The treatment group also received HBOT 5 days per week for 4 weeks, for a total of 20 treatment sessions. Each HBOT session lasted 120 minutes at 2.4 ATA. Measures of HS Severity Index (HSSI), Sartorius score (SS), Dermatology Life Quality Index (DLQI), visual analog scale (VAS) for pain, erythrocyte sedimentation rate (ESR), and C-reactive protein (CRP) were used to assess HS (13). Yildiz et al. found that HBOT served as an efficacious adjunctive therapy in the antibiotic treatment of HS. Treatment efficacy in the HBOT group was superior compared to the control group $(p<0.05)$. At the end of weeks 4 and 10, there were statistically significant improvements in SS (week 4 and 10, $p=0.021$ ), HSSI (week 4 and 10, $p=0.009$ ), and DLQI (week 4, $p=0.044$; week 10, $p=0.009$ ) (13). By the end of week 10, statistically significant improvements were demonstrated in ESR $(p=0.048)$ and VAS $(p=0.009)$. However, there was no statistically significant improvement in CRP at weeks 4 or 10 (13).

Only one case of HS that was unresponsive to HBOT has been reported in the literature (14). The patient suffered from extensive disease for more than 20 years and had failed treatment with multiple wide excisions, several fistulotomies, HBOT, and a diverting colostomy. The patient eventually responded to a combined approach of surgery and perilesional injections of granulocyte-macrophage colony-stimulating factor (14).

\section{Pyoderma gangrenosum}

Several case reports describing the use of HBOT in the treatment of pyoderma gangrenosum have been published. Barr et al. were the first to report the successful treatment of pyoderma gangrenosum with HBOT in 1972 (15). In 1992, Wasserteil et al. published a case of a middle-aged woman with uncontrolled diabetes mellitus and severe pyoderma gangrenosum on both legs that was refractory to standard treatments (16). A trial of HBOT was attempted: the patient spent 90 minutes daily in a hyperbaric chamber set at 2.4 ATA. Within a few days, there was noticeable improvement in the appearance of her leg ulcers. In addition, the patient had significant pain relief. The patient's prednisone was tapered from 80 mg daily down to $10 \mathrm{mg}$ daily, allowing better blood glucose control. After 33 HBOT treatments, the patient's ulcers on the left leg completely resolved. After 68 treatments, the ulcers on her right leg had almost completely healed and her left leg ulcers remained clear. Within 1 month, the ulcers on the right leg resolved, and at a 4-month follow-up visit there was no recurrence (16).

Niezgoda et al. reported a case of a middle-aged man with an expanding pyoderma gangrenosum leg ulcer that had been refractory to standard treatment (17). The patient was started on HBOT at 2.4 ATA while continuing his dose of prednisone $60 \mathrm{mg}$ daily. After just 2 days of HBOT, the ulcer stabilized in size. At that point, wide surgical debridement was completed followed by split-thickness skin grafting. Vacuum-assisted closure and HBOT were continued after skin grafting for a total of 11 HBOT treatments. He was discharged on a tapered dose of prednisone and instructions for local wound care. At outpatient follow-up visits, the patient had no evidence of recurrence (17). A case report by Vieira et al. published in 2011 described an adolescent patient that responded well to HBOT in combination with corticosteroids and immunosuppressant therapy (18). The patient had been on prednisone $40 \mathrm{mg}$ daily and cyclosporine $200 \mathrm{mg}$ daily for the preceding 8 months, but her leg ulcers continued to worsen. She had an acute exacerbation of her condition after losing coverage of cyclosporine for 1 month. She was admitted and underwent 10 90-minute HBOT sessions at 2.5 ATA, with an excellent response (18).

In 2007, Tutrone et al. published a retrospective review examining the use of HBOT in the treatment of pyoderma gangrenosum (19). Of the 15 cases they reviewed, 11 demonstrated a positive response to HBOT therapy, and only four cases resulted in no therapeutic benefit (19). In 2015, Cabalag et al. published a retrospective review of the inpatient management of pyoderma gangrenosum (20). Of the 29 patients included in the study, eight (28\%) received HBOT. Of these eight patients, one demonstrated complete heal- 
ing of his/her ulcer, five had improvement but not complete healing of their ulcers, one had stabilization of his/her ulcer, and one had worsening of ulcers after discharge (20). A study published by Feitosa et al. in 2016 investigated the effect of HBOT in patients with refractory Crohn's disease (21). The endpoint of the study was complete healing of pyoderma gangrenosum and perianal disease and complete closure of enterocutaneous fistulas. The HBOT sessions were performed at 2.4 ATA and each session lasted for 2 hours. Of the 29 patients included in the study, five had pyoderma gangrenosum. One patient had both pyoderma gangrenosum and enterocutaneous fistulas, and one patient had pyoderma gangrenosum, enterocutaneous fistulas, and perianal Crohn's disease. These five patients received an average of 31.2 HBOT sessions, and all five patients demonstrated a satisfactory response to HBOT therapy. None of the five patients experienced adverse side effects (21).

Our search of the literature revealed only two reported cases in which HBOT did not result in improvement of pyoderma gangrenosum (22-23). One was an atypical case of bullous pyoderma

\section{References}

1. Weaver, Lindell K. Hyperbaric oxygen therapy indications, 13th ed. North Palm Beach, FL: Best Publishing Company; 2014.

2. Behnke, Albert R. Hyperbaric oxygenation. N Engl J Med. 1967;276:1423-9.

3. Neuman TS, Thom SR. Physiology and medicine of hyperbaric oxygen therapy. Philadelphia: Elsevier; 2008.

4. Wilkinson D, Doolette D. Hyperbaric oxygen treatment and survival from necrotizing soft tissue infection. Arch Surg. 2004;139:1339-45.

5. George ME, Rueth NM, Skarda DE, Chipman JG, Quickel RR, Beilman GJ. Hyperbaric oxygen does not improve outcome in patients with necrotizing soft tissue infection. Surg Infect (Larchmt). 2009;10:21-8.

6. Levett D, Bennet MH, Millar I. Adjunctive hyperbaric oxygen for necrotizing fasciitis. Cochrane Database Syst Rev. 2015;1:CDo07937.

7. Heyboer M, Sharma D, Santiago W, McCulloch N. Hyperbaric oxygen therapy: side effects defined and quantified. Adv Wound Care (New Rochelle). 2017;6: 210-24.

8. Francis A, Baynosa RC. Hyperbaric oxygen therapy for the compromised graft or flap. Adv Wound Care. 2017;6:23-32.

9. Larson JV, Steensma EA, Flikkema RM, Norman EM. The application of hyperbaric oxygen therapy in the management of compromised flaps. Undersea Hyperb Med. 2013;40:499-504.

10. Skeik N, Porten BR, Isaacson E, Seong J, Klosterman DL, Garberich RF, et al. Hyperbaric oxygen treatment outcome for different indications from a single center. Ann Vasc Surg. 2015;29:206-14.

11. Goldman RJ. Hyperbaric oxygen therapy for wound healing and limb salvage: a systematic review. PM R. 2009;1:471-89.

12. Ozdemir Y, Uzun G, Mutluoglu M, Gulec B. Hyperbaric oxygen therapy for the management of postsurgical wounds in hidradenitis suppurativa. Am Surg. 2010;76:E237-8.

13. Yildiz H, Senol L, Ercan E, Bilgili ME, Karabudak AO. A prospective randomized controlled trial assessing the efficacy of adjunctive hyperbaric oxygen therapy in the treatment of hidradenitis suppurativa. Int J Dermatol. 2016;55:232-7.

14. Sharon-Guidetti A, Ziv Y, Kummer E, Yogev R, Halevy A. Granulocyte-macrophage colony-stimulating factor for perianal hidradenitis suppurativa: report of a case. Dis Colon Rectum. 2006;49:682-4.

15. Barr PO, Enforts W, Eriksson G. Hyperbaric oxygen therapy in dermatology. Br J Dermatol. 1972;86:631-5.

16. Wasserteil V, Bruce S, Sessoms SL, Guntupalli KK. Pyoderma gangrenosum treated with hyperbaric oxygen therapy. Int J Dermatol. 1992;31:594-6. gangrenosum that did not respond to 15 sessions of HBOT (22). The second case described a young man that had undergone a wide variety of treatments, including oral prednisone, two skin grafts, dapsone, methotrexate, pulse methylprednisolone therapy, and HBOT, among other therapeutic approaches (23). The authors did not specify the number of HBOT sessions performed. Ultimately, the patient was only responsive to the chemotherapy agent chlorambucil (23).

\section{Conclusion}

Overall, the case reports and reviews summarized in this paper tend to report positive results with the use of HBOT as an adjunctive treatment for NSTIs, compromised grafts and flaps, HS, and pyoderma gangrenosum. However, there is an evident need for high-quality randomized controlled trials to confirm these encouraging findings, especially given that HBOT is not without risk. In addition, whether HBOT could offer any utility as a standalone treatment for these conditions is yet to be determined.
17. Niezgoda JA, Cabigas EB, Allen HK, Simanonok JP, Kindwall EP, Krumenauer J. Managing pyoderma gangrenosum: a synergistic approach combining surgical debridement, vacuum-assisted closure, and hyperbaric oxygen therapy. Plast Reconstr Surg. 2006;117:24e-8e.

18. Vieira WA, Barbosa LR, Martin LM. Hyperbaric oxygen therapy as an adjuvant treatment for pyoderma gangrenosum. An Bras Dermatol. 2011;86:1193-6.

19. Tutrone WD, Green K, Weinberg JM, Caglar S, Clarke D. Pyoderma gangrenosum: dermatologic application of hyperbaric oxygen therapy. J Drugs Dermatol. 2007;6:1214-9.

20. Cabalag MS, Wasiak J, Lim SW, Raiola FB. Inpatient management of pyoderma gangrenosum: treatments, outcomes, and clinical implications. Ann Plast Surg. 2015;74:354-60.

21. Feitosa MR, Féres FO, Tamaki CM, Perazzoli C, Bernardes MV, Parra RS, et al. Adjunctive hyperbaric oxygen therapy promotes successful healing in patients with refractory Crohn's disease. Acta Cir Bras. 2016;31:19-23.

22. Altunay IK, Sezgin SA, Ileri U, Ekmekçi TR, Kuran I, Köslü A, et al. Atypical hemorrhagic bullous pyoderma gangrenosum. Int J Dermatol. 2001;40:327-9.

23. Callen JP, Case JD, Sager D. Chlorambucil-an effective corticosteroid-sparing therapy for pyoderma gangrenosum. J Am Acad Dermatol. 1989;21:515-9.

24. Darling MD, Peterson JD, Fabi SG. Impending necrosis after injection of hyaluronic acid and calcium hydroxylapatite fillers: report of 2 cases treated with hyperbaric oxygen therapy. Dermatol Surg. 2014;40:1049-52.

25. Juan WH, Chan YS, Lee JC, Yang LC, Hong HS, Yang CH. Livedoid vasculopathy: long-term follow-up results following hyperbaric oxygen therapy. $\mathrm{Br} J$ Dermatol. 2006;154:251-5.

26. Meiss F, Marsch WC, Fischer M. Livedoid vasculopathy. The role of hyperhomocysteinemia and its simple therapeutic consequences. Eur J Dermatol. 2006;16: $159-62$.

27. Tutrone WD, Green KM, Norris T, Weinberg JM, Clarke D. Brown recluse spider envenomation: dermatologic application of hyperbaric oxygen therapy. J Drugs Dermatol. 2005;4:424-8.

28. Swanson DL, Vetter RS. Bites of brown recluse spiders and suspected necrotic arachnidism. N Engl J Med. 2005;352:700-7.

29. Sylva DE, Nangpal S, John MJ, Shah KM, Bhattacharya S, Mani H, et al. Therapeutic hyperbaric oxygenation as an adjunct to multidrug therapy in Hansen's disease. Med J Armed Forces India. 1999;55:98-103. 